\title{
Development of Graphene Based Inks for Deposition via Inkjet Printing for Sensing Application ${ }^{\dagger}$
}

\author{
Caterina Travan ${ }^{1,2, *}$, Martin Mischitz ${ }^{1}$, Alexander Zöpfl ${ }^{3}$, Ulrich Krumbein ${ }^{4}$ and \\ Prashant Makaram ${ }^{4}$ \\ 1 Infineon Technologies Austria AG, Siemensstraße 2, 9500 Villach, Austria; martin.mischitz@infineon.com \\ 2 Institute of Electronic Sensor System, Technische Universität Graz, 8010 Graz, Austria \\ 3 Infineon Technologies AG, Wernerwerkstraße 2, 93049 Regensburg, Germany; \\ alexander.zoepfl@infineon.com \\ 4 Infineon Technologies AG, Am Campeon 1-15, 85579 Neubiberg, Germany; \\ ulrich.krumbein@infineon.com (U.K.); prashant.makaram@infineon.com (P.M.) \\ * Correspondence: caterina.travan@infineon.com; Tel.: +49-170-2896408 \\ † Presented at the Eurosensors 2018 Conference, Graz, Austria, 9-12 September 2018.
}

Published: 17 December 2018

\begin{abstract}
In this work we investigate and optimize graphene based inks to achieve a stable and wellcontrollable jetting behavior using a DoD (Drop on Demand) inject printer which has all the required characteristics of a tool for mass production.
\end{abstract}

Keywords: graphene; inkjet printing; inks; gas sensors

\section{Introduction}

Graphene is a 2D material which shows excellent sensor properties thanks to its very high surface area and carrier mobility and offers the possibility to develop very sensitive, cheap and low power consumption gas sensors [1]. In order to achieve the highest sensitivity, it is important to deposit a well-structured thin film of graphene on the electrode. Inkjet printing is a widely used technique in printed electronics which has the potential to produce such film in a stable and reproducible way. However the commercially available graphene big agglomerations of the flakes and high percentage of different binders which influence the sensing properties. Additionally despite many graphene suppliers are present on the market using different methods to exfoliate or grow the graphene flakes, just a few of them are actually able to produce large amounts of high quality graphene in a stable and reproducible way [2]. Therefore several challenges are related with the development of a suitable graphene ink for mass production of gas sensing device: (a) selection of high purity few layer graphene material; (b) rheological properties and surface tension of the fluid leading to an optimal drop formation and jetting performance with productive printhead systems; (c) long shelf time with low amount of addictive; (d) the spreading of the ink on the surface must lead to on homogeneous film formation without coffee stain effect.

\section{Materials and Methods}

Since none of the commercially available graphene based inks showed good printing properties and homogeneous drying after deposition, the 30 different graphene inks which have been tested have been partially developed by Infineon itself and partially by external companies. The inks differ from each other for the main solvent used (e.g., different type of alcohols and organic solvents), the type and amount of additives, the concentration and the producer of the graphene flakes. The printing behavior of theses inks has been tested using a Pixdro LP50 (Meyer Burger, Gwatt, 
Switzerland) inkjet printer (produced by Meyer Burger) with a Dimatix SE128AA (Fujifilm, Santa Clara, USA) printhead. This printhead has 128 nozzles and can be used for mass production but, unlike disposable cartridge used in laboratories, it is not suitable for printing water base inks, therefore the need to develop water free graphene inks. The printing performance were compared with the ones predicted by theory using the dimentionless numbers Reynolds, Weber and Ohnesorge [3,4]. Advanced Drop Analysis, a software developed by Meyer Burger, was used to extract information about droplet size, speed and angel from the normal trajectory from the drop view camera. Raman spectroscopy has been used to compare the graphene flakes used in the different inks in terms of thickness and amount of defects and combine these results with the optical investigation with SEM, which provides information about lateral flake size and thickness. Raman spectra have been measured at CTR using an equipment produced by Renishaw with a laser wavelength of 532 $\mathrm{nm}$, a laser aperture of $50 \%$ and an exposure time of $1 \mathrm{~s}$. The SEM used was Hitachi SU-70.

\section{Results and Discussion}

Figure 1 shows the calculated Reynolds and Weber numbers of some of the investigated inks, just the latest developed ones are in the window of optimal jetting performance. All the inks outside the white area of the graph showed either no jetting or very poor one, i.e., very slow droplet, presence of satellites and high deviation from $90^{\circ}$ angle with the substrate. The inks inside this area exhibited in most of the case good fine droplet formation, but not always. The comparison between printing performance and predicted ones using dimensionless numbers revealed some weakness in this prediction model due to non Newtonian characteristics of most of the inks, e.g., two inks with very similar values of surface tension, viscosity in the optimal windows have manifested in the empirical tests in one case fine jetting performance and in the other on drop formation. Further analysis revealed that the first ink was almost a Newtonian fluid, while the second one had a strong non Newtonian shear thickening rheopectic behavior. Since the shear rate inside the printhead is very high those results explains the absence of jetting despite the theory predicted an optimal droplet formation. The combination of theory and empirical results led to the optimization of the ink formulation. The homogeneous distribution of the flakes during drying have been achieved using surface engineering and fine tuning of the inks.

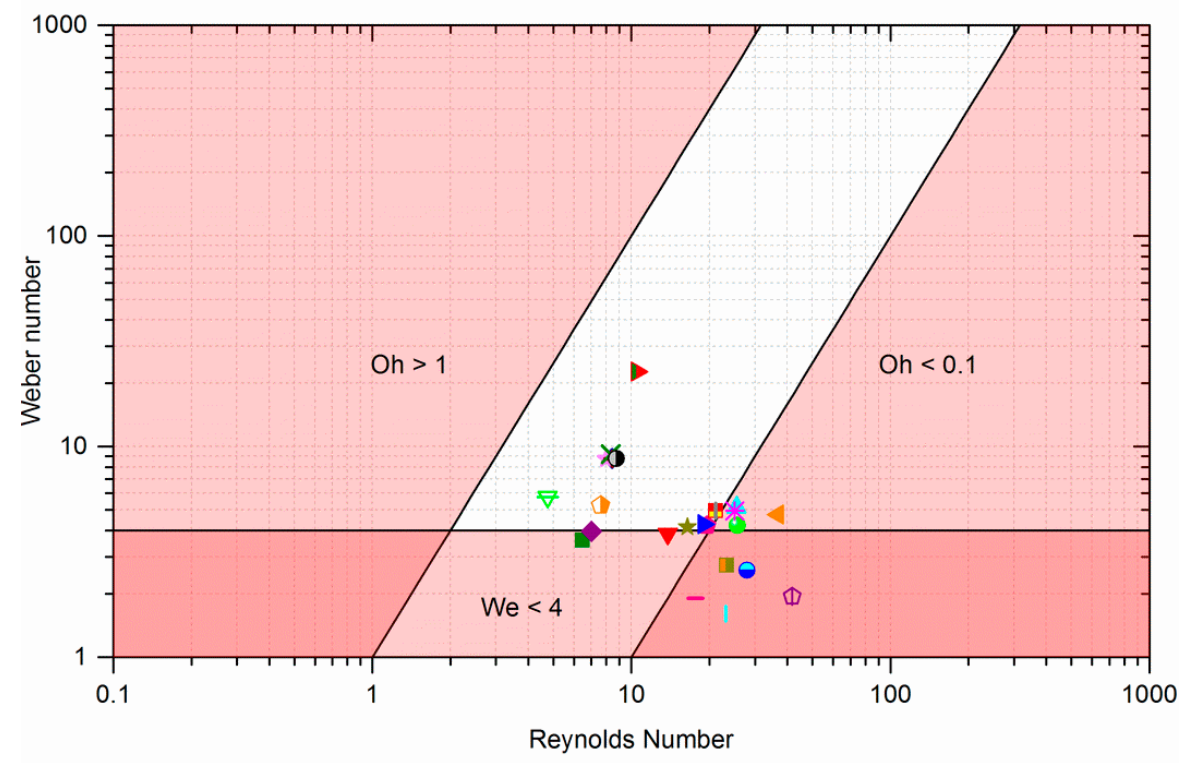

Figure 1. Plot which shows the operating window for a stable printing (white area) base on the values of the Reynolds and Weber numbers. The values of some of the investigated graphene inks are displayed in the graph. 
The graphene flakes used were produced with different method showing different Raman spectra (Figure 2a). The flakes produced via electrochemical exfoliation have high D and $\mathrm{G}$ and almost no 2D peak, revealing high amount of defects and multi-layers. The $G$ peak is shifted and broaden indicating defects and a certain amount of oxidation. The graphene produced with a patented plasma process have lower $\mathrm{D}$ peak and higher 2D peak, the $\mathrm{G}$ peak is narrower compared to the other material. The second material has therefore a lower amount of defects and probably consists of mono-bilayer graphene [5].

The SEM analysis (Figure 2b,c) shows the difference in lateral size and adhesion on the surface of these two type of graphene flakes.

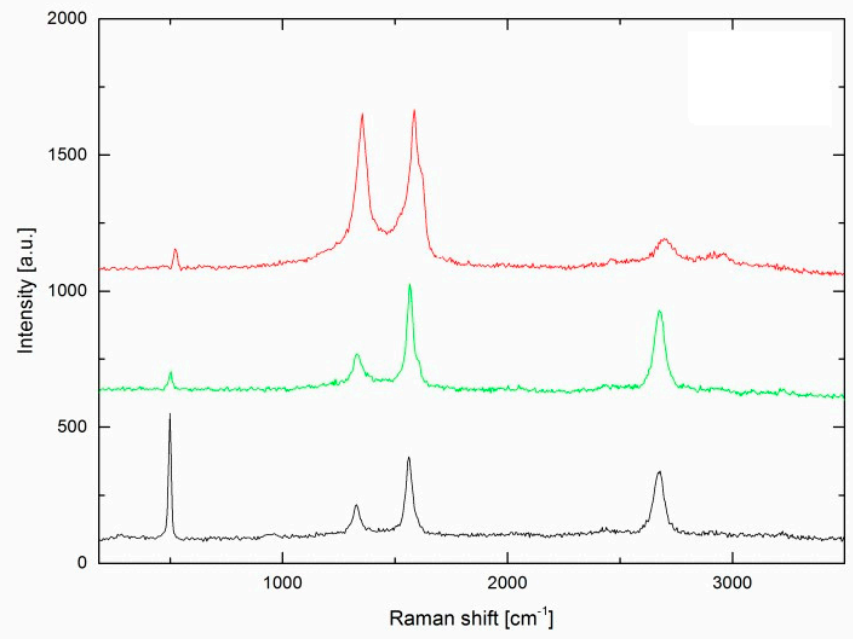

(a)

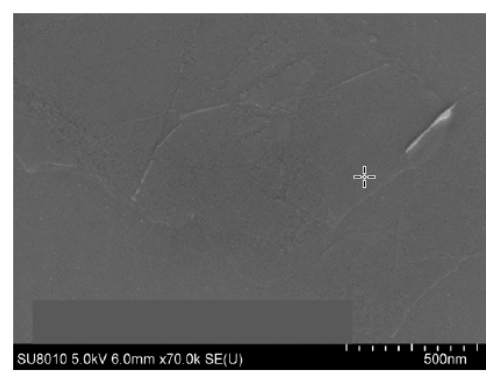

(b)

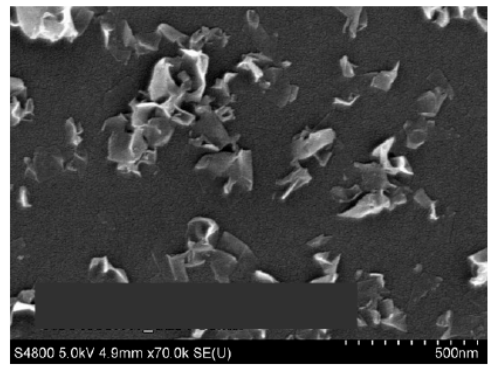

(c)

Figure 2. (a) Raman spectra of three different graphene flakes deposited using inkjet printing. The curve in red is the Raman spectrum of graphene flakes produced via electrochemical exfoliation. The curves in green and black are the Raman spectra of mono/two layers of graphene produced with a patented plasma process. (b,c) SEM pictures of graphene flakes produced with different methods: (b) few $\mu \mathrm{m}$ later size and few layer graphene produced by electrochemical exfoliation; (c) SEM picture of graphene flakes with small lateral size and mono/two layers of graphene produced with a patented plasma process.

\section{Conclusions}

The reported investigation brought to the development of a graphene based ink with an optimal jetting performance using a inkjet printing system suitable for mass production. The graphene flakes used in theses inks have been selected based on their purity and thickness.

Author Contributions: All authors were involved in the design of the experiments. C.T. performed the experiments and wrote the paper.

Acknowledgments: The Raman spectra present in this paper were performed by CTR in Villach.

Conflicts of interest: the authors declare no conflict of interest.

\section{References}

1. Llobet, E. Gas sensors using carbon nanomaterials: A review. Sens. Actuators B Chem. 2013, 179, 32-45, doi:10.1016/j.snb.2012.11.014.

2. Ghaffarzadeh, K. Graphene, 2D Materails and Carbon Nanotubes: Markets, Technologies and Opportunities 20162026; IDTechEx: Cambridge, UK, 2016. 
3. Hutchings, I.M.; Martin, G.D. Martin, Inkjet Technology for Digital Fabrication, 1st ed.; John Wiley \& Sons: Hoboken, NJ, USA, 2013.

4. Derby, B. Inkjet printing of Functional and Structural Materails: Fluid Property Requirements, Feature Stability and Resolution. Annu. Rev. Mater. Res. 2010, 40, 395-414, doi:10.1146/annurev-matsci-070909104502.

5. Eckmann, A.; Felten, A.; Mishchenko, A.; Britnell, L.; Krupke, R.; Novoselov, K.S.; Casiraghi, C. Probing the nature of Defects in Graphene by Raman Spectroscopy. Nano Lett. 2012, 12, 3925-3930, doi:10.1021/nl300901a.

(C) 2018 by the authors. Licensee MDPI, Basel, Switzerland. This article is an open access article distributed under the terms and conditions of the Creative Commons Attribution (CC BY) license (http://creativecommons.org/licenses/by/4.0/). 